\title{
FIRST ORDER POINCARÉ INEQUALITIES IN METRIC MEASURE SPACES
}

\author{
Estibalitz Durand-Cartagena, Jesús A. Jaramillo \\ and Nageswari Shanmugalingam
}

UNED, ETS de Ingenieros Industriales, Departamento de Matemática Aplicada 28040-Madrid, Spain; edurand@ind.uned.es

Universidad Complutense de Madrid, Departamento de Análisis Matemático 28040-Madrid, Spain; jaramil@mat.ucm.es

University of Cincinnati, Department of Mathematical Sciences

P.O. Box 210025, Cincinnati, OH 45221-0025, U.S.A.; shanmun@ucmail.uc.edu

\begin{abstract}
We study a generalization of classical Poincaré inequalities, and study conditions that link such an inequality with the first order calculus of functions in the metric measure space setting when the measure is doubling and the metric is complete. The first order calculus considered in this paper is based on the approach of the upper gradient notion of Heinonen and Koskela [HeKo]. We show that under a Vitali type condition on the BMO-Poincaré type inequality of Franchi, Pérez and Wheeden [FPW], the metric measure space should also support a $p$-Poincaré inequality for some $1 \leq p<\infty$, and that under weaker assumptions, the metric measure space supports an $\infty$-Poincaré inequality in the sense of [DJS].
\end{abstract}

\section{Introduction}

The Poincaré inequality is one of the fundamental inequalities in harmonic analysis and the theory of partial differential equations; it states that there exists a constant $C>0$ such that the inequality

$$
f_{B}\left|f-f_{B}\right| d \mathscr{L}^{n} \leq C r f_{B}|\nabla f| d \mathscr{L}^{n}
$$

where $B$ is a ball of radius $r$ and $f_{B}:=\mu(B)^{-1} \int_{B} f d \mu$ is the average of $f$ over $B$, holds for each ball $B$ in the Euclidean space and all functions $f \in W^{1,1}(B)$. There have been many generalizations of Poincaré inequalities. The inequality

$$
f_{B}\left|f-f_{B}\right| d \mathscr{L}^{n} \leq C r\left(f_{B}|\nabla f|^{p} d \mathscr{L}^{n}\right)^{1 / p}
$$

is called a $p$-Poincaré inequality. It is important to notice that the exponent $p$ plays a role. For example, there are Euclidean domains which satisfy a $p$-Poincaré inequality for sufficiently large $p$ but not for $p=1$.

In the more general setting of metric measure spaces $(X, d, \mu)$ (spaces equipped with a metric $d$ and a Borel regular measure $\mu$ ), Heinonen and Koskela [HeKo] introduced the notion of "upper gradients" which serves the role of (modulus) of derivatives; one can use this notion to make sense of the right-hand side term of the Poincaré

doi:10.5186/aasfm.2013.3825

2010 Mathematics Subject Classification: Primary 31E05, 30L99, 43A85.

Key words: Poincaré inequality, BMO-Poincaré inequality, quasiconvexity, Lipschitz functions, Newtonian functions, thick quasiconvexity.

The first and second authors are partially supported by grant MTM2009-07848 (Spain). The third author is partially supported by grant \# 200474 from the Simons Foundation (USA). 
inequalities in this a priori non-linear setting (see Definition 2.3). However, there are other alternative notions of Sobolev classes of functions in the metric setting, with their competing notions of Poincaré inequalities; see for example [Sh2] and [KST]. It is therefore natural to consider a generalization of these various notions of Poincaré inequalities.

One natural generalization consists of replacing the right-hand side of the inequality by a more general functional defined over the class $\mathcal{B}$ of all balls in $X$. Namely, for each function $f$ in a class $\mathcal{F}$ we consider a functional $a_{f}: \mathcal{B} \rightarrow[0, \infty)$ such that whenever $B \in \mathcal{B}$,

$$
f_{B}\left|f-f_{B}\right| d \mu \leq a_{f}(B)
$$

These types of functionals, satisfying certain additional conditions, were first considered in [FPW] and further studied in [LP], [MP1, MP2], [OP], [BJM], and the references therein. In [HKT] Sobolev-type spaces were studied by means of this generalized Poincare inequalities, with the additional assumption that the functional $a_{f}$ is given by a Radon measure $\nu_{f}$. On the other hand, in [ABKY] BMO-type functions defined by John and Nirenberg were studied in the setting of metric measure spaces. Our aim in this paper is to establish that, under mild geometric conditions on the functional $a_{f}$ that are different from that of [FPW] (see Definition 3.1), inequality (1) encodes geometric information of the metric measure space and that it implies the Poincaré inequalities associated with the upper gradient structure of Heinonen and Koskela.

In the context of spaces of homogeneous type (complete metric measure spaces equipped with a doubling measure), [FPW] presents a general method based on the Calderón-Zygmund theory and the good- $\lambda$ inequalities to establish, under some conditions on the functional $a_{f}$ (see Definition 2.5), that the left-hand side of the inequality (1) can be self-improved (see Theorem 2.6).

One can consider simple functionals to illustrate that the conditions imposed to the functional in [FPW] are associated with $L^{p}$-theory rather than first order calculus. For example, for a family of functions $\mathcal{F}=L^{p}(X)$ one can consider the functional given by the left hand side of the Poincaré inequality

$$
a_{f}(B)=\left(f_{B}\left|f-f_{B}\right|^{p} d \mu\right)^{1 / p} .
$$

which satisfies all the conditions in Definition 2.5 but would certainly be more related to $L^{p}$-theory. Therefore, without any additional conditions on the functional $f \mapsto a_{f}$, any self-improvement properties of the generalized Poincaré inequality are related to the $L^{p}$-theory, and are associated with the self-improvement of the left-hand side term of the inequality. Hence further conditions are needed in order to obtain information relevant to first order calculus and geometry of metric spaces.

On the other hand, recently Keith and Zhong [KZ] proved a self-improving property for Poincaré inequalities, that is, if $X$ is a complete metric space equipped with a doubling measure satisfying a $p$-Poincaré inequality for some $1<p<\infty$, then there exists $\varepsilon>0$ such that $X$ supports a $q$-Poincaré inequality for all $q>p-\varepsilon$. The result of [KZ] depends heavily on the fact that the right-hand side of the Poincare inequality is given via an integral of the pointwise Lipschitz constant function Lip $f$ for Lipschitz functions $f$; more importantly, it is given in terms of an integral of a 
function, and so it is clear what is meant by the self-improvement of the right-hand side. The second ingredient used in the proof of [KZ] is the fact that the functional $f \mapsto a_{f}$, given via a Sobolev energy, satisfies a stronger truncation property than the truncation property (4). In particular, they need the fact that the Sobolev energy of a function that is Lipschitz continuous on a set can be controlled on that set by its Lipschitz constant. These two characteristics of the proof of [KZ] gives us a hint on what conditions we should look for in the functional $f \mapsto a_{f}$ that links the generalized Poincaré inequality to a real first order calculus.

In the present work we study geometric and analytical properties of the functional $\mathcal{F} \ni f \mapsto a_{f}$ that guarantee that the $a_{f}$ are indeed related to the Sobolev energy rather than to $L^{p}$-energy. We consider the choice of $\mathcal{F}=N^{1, \infty}(X)$, the NewtonianSobolev space of $\infty$-type considered in [DJ] and [DJS]; however, much of the results also hold when $\mathcal{F}$ is the class of all bounded Lipschitz functions on $X$, under some additional mild geometric hypotheses on $X$; we will point these results out in remarks along this note.

The paper is organized as follows. In Section 2 we give an exposition of the notions of upper gradients, $p$-Poincaré inequalities, and the geometric notion of $p$ thick quasiconvexity of a metric measure space. We also discuss here the conditions imposed to the functional in [FPW] and illustrate throughout examples that these conditions by themselves, are not an indicative of first order calculus but are more associated with the $L^{p}$-theory.

In Section 3 we propose conditions on the functional $f \mapsto a_{f}$ (Definition 3.2) to link the generalized Poincaré inequality with first order calculus, and show that some of these conditions imply versions of quasiconvexity of the metric space. We also give some examples in this section to illustrate these conditions.

In Section 4 we study the links between the generalized Poincaré inequality and the $p$-Poincaré inequality that is associated with the upper gradient structure. In the main result of this paper, Theorem 4.1, we show that if the functional $f \mapsto a_{f}$, associated with the generalized Poincaré inequality, satisfies the all the conditions given in Definition 3.2, then the metric space also supports a $p$-Poincare inequality (for the index $p$ associated with Condition $\left(V_{p}\right)$ of Definition 3.2). We also show that if the functional $f \mapsto a_{f}$ is associated with a Radon measure as in [HKT], then for each Lipschitz function $f$ the functional $a_{f}$ is associated to the integral average of an upper gradient of $f$; see Proposition 4.4. The second main result of this paper, Theorem 4.5, also appears in Section 4; it shows that when the functional $f \mapsto a_{f}$ satisfies one of the conditions given in Definition 3.2, Condition (iii), then the metric space also supports an $\infty$-Poincaré inequality associated with the upper gradient structure.

Acknowledgement. We wish to thank Rafael Espínola Garcia and Carlos Pérez for their encouragement and for the questions that motivated the research of this paper, and the two anonymous referees whose suggestions significantly helped to improve the exposition of the paper. This research began during the visit of the third author to Universidad Complutense de Madrid, and continued during the time the first and third authors visited Universidad de Sevilla; they wish to thank those institutions for their kind hospitality. 


\section{Notation and preliminaries}

We assume that $(X, d, \mu)$ is a metric measure space, that is, a metric space equipped with a metric $d$ and a Borel measure $\mu$ such that $0<\mu(B)<\infty$ for each open ball $B \subset X$. We assume in addition that $\mu$ is doubling, that is, there is a constant $C_{\mu}>0$ such that for all $x \in X$ and $r>0$,

$$
\mu(B(x, 2 r)) \leq C_{\mu} \mu(B(x, r)) .
$$

In the above definition of doubling measure, we can equivalently replace open balls $B(x, R)$ with closed balls $\bar{B}(x, R)=\{y \in X: d(y, x) \leq R\}$, at the penalty of a larger constant $C_{\mu}$.

By a path $\gamma$ we mean a continuous mapping $\gamma:[a, b] \rightarrow X$. Recall that the length of a path $\gamma:[a, b] \rightarrow X$ is given by

$$
\ell(\gamma)=\sup \left\{\sum_{i=0}^{n-1} d\left(\gamma\left(t_{i}\right), \gamma\left(t_{i+1}\right)\right)\right\}
$$

where the supremum is taken over all finite partitions $a=t_{0}<t_{1}<\cdots<t_{n}=b$ of the interval $[a, b]$. We say that a path $\gamma$ is rectifiable if $\ell(\gamma)<\infty$. The integral of a Borel function $g$ over a rectifiable path $\gamma$ is defined via the arc-length parametrization $\gamma_{0}$ of $\gamma$ as follows:

$$
\int_{\gamma} g d s=\int_{0}^{\ell(\gamma)} g \circ \gamma_{0}(t) d t .
$$

Recall here that every rectifiable path $\gamma$ admits a parametrization by arc-length; that is, with $\gamma_{0}:[a, b] \rightarrow X$, for all $t_{1}, t_{2} \in[a, b]$ with $t_{1} \leq t_{2}$, we have $\ell\left(\gamma_{\left.0\right|_{\left[t_{1}, t_{2}\right]}}\right)=t_{2}-t_{1}$. Hence from now on we only consider paths that are arc-length parametrized. We say that a metric space $X$ is quasiconvex if there is a constant $C \geq 1$ such that for each pair of points $x, y \in X$ there is a curve $\gamma$, with endpoints $x$ and $y$, such that $\ell(\gamma) \leq C d(x, y)$. We also say that $X$ is a geodesic space if it is quasiconvex with constant $C=1$.

We next recall the definition of $p$-modulus, an outer measure on the collection of all paths in $X$.

Definition 2.1. (Modulus of a family of paths) Let $\Gamma$ be a family of non-constant rectifiable paths in $X$. For $1 \leq p \leq \infty$ we define the $p$-modulus of $\Gamma$ by

$$
\operatorname{Mod}_{p}(\Gamma)= \begin{cases}\inf _{g} \int_{X} g^{p} d \mu & \text { if } 1 \leq p<\infty \\ \inf _{g}\|g\|_{L^{\infty}(X)} & \text { if } p=\infty\end{cases}
$$

where the infimum is taken over all non-negative Borel functions $g: X \rightarrow[0, \infty]$ such that $\int_{\gamma} g d s \geq 1$ for all $\gamma \in \Gamma$. If a property holds for all rectifiable paths in $X$ except for a family $\Gamma$ with $\operatorname{Mod}_{p} \Gamma=0$, then we say that the property holds for $p$-a.e. path.

A useful generalization of Sobolev spaces to general metric spaces is the Newtonian Spaces $N^{1, p}(X)$ introduced in [Sh, Sh1]. The space $N^{1, \infty}(X)$ was introduced and studied in [DJ]. The definition is based on the notion of upper gradients of Heinonen and Koskela [HeKo] and weak upper gradients of Koskela and MacManus [KoMc]. 
Definition 2.2. A non-negative Borel function $g$ on $X$ is a $p$-weak upper gradient of an extended real-valued function $f$ on $X$ if

$$
|f(\gamma(a))-f(\gamma(b))| \leq \int_{\gamma} g d s
$$

for $p$-a.e. rectifiable path $\gamma$ in $X$. We interpret the above inequality as also requiring that $\int_{\gamma} g d s=\infty$ whenever at least one of $f(\gamma(a)), f(\gamma(b))$ is not finite. We say that a $p$-weak upper gradient $g$ is an upper gradient if the above inequality holds for each rectifiable curve $\gamma$ on $X$.

For $1 \leq p \leq \infty$, let $\widetilde{N}^{1, p}(X, d, \mu)$ be the class of all $p$-integrable functions on $X$ that have a $p$-weak upper gradient in $L^{p}(X)$. For $f \in \widetilde{N}^{1, p}(X, d, \mu)$ we define

$$
\|f\|_{N^{1, p}}:=\|f\|_{L^{p}(X)}+\inf _{g}\|g\|_{L^{p}(X)}
$$

where the infimum is taken over all $p$-weak upper gradients $g$ of $f$. Now, we define in $\widetilde{N}^{1, p}(X, d, \mu)$ an equivalence relation by $f_{1} \sim f_{2}$ if and only if $\left\|f_{1}-f_{2}\right\|_{N^{1, p}}=0$. Then the space $N^{1, p}(X, d, \mu)=N^{1, p}(X)$ is defined as the quotient $\widetilde{N}^{1, p}(X, d, \mu) / \sim$ and it is equipped with the norm $\|f\|_{N^{1, p}(X)}:=\|f\|_{N^{1, p}}$.

The following Poincaré inequality is now standard in literature on analysis on metric spaces. It was first formulated in [HeKo] for $1 \leq p<\infty$. The case $p=\infty$ was introduced in [DJS].

Definition 2.3. Let $1 \leq p \leq \infty$. We say that $(X, d, \mu)$ supports a weak $p$ Poincaré inequality if there are constants $\lambda_{p}, C_{p}>0$ such that when $f: X \rightarrow \mathbf{R} \cup$ $\{-\infty, \infty\}$ is a measurable function, $g: X \rightarrow[0, \infty]$ an upper gradient of $f$, and $B(x, r)$ a ball in $X$, then

$$
f_{B(x, r)}\left|f-f_{B(x, r)}\right| d \mu \leq C_{p} r\left(f_{B\left(x, \lambda_{p} r\right)} g^{p} d \mu\right)^{1 / p}
$$

if $1 \leq p<\infty$, and

$$
f_{B(x, r)}\left|f-f_{B(x, r)}\right| d \mu \leq C_{\infty} r\|g\|_{L^{\infty}\left(B\left(x, \lambda_{\infty} r\right)\right)}
$$

if $p=\infty$. Note that it is necessary to have $\lambda_{p} \geq 1$. The word weak refers to the possibility that $\lambda_{p}$ may be larger than 1 . Since the constant $\lambda_{p}$ does not play a significant role in this paper (recall that the measure $\mu$ is doubling on $X$ ), and so in subsequent reference to this inequality in this paper we will suppress the term "weak". Here for measurable sets $A \subset X$ with $0<\mu(A)<\infty$ we write

$$
f_{A}=f_{A} f:=\frac{1}{\mu(A)} \int_{A} f d \mu .
$$

Recall the definition of $\operatorname{Mod}_{p}(\Gamma)$ for a family $\Gamma$ of paths in $X$ from Definition 2.1.

Definition 2.4. Fix $1 \leq p \leq \infty$. We say that $X$ is $p$-thick quasiconvex if there is a constant $C \geq 1$ such that whenever $x, y \in X$ with $x \neq y$ and $0<\varepsilon<d(x, y) / 4$, and whenever $E \subset B(x, \varepsilon)$ and $F \subset B(y, \varepsilon)$ are measurable sets with $\mu(E) \mu(F)>0$, with $\Gamma$ denoting the collection of all paths $\gamma$ in $X$ connecting $E$ to $F$ with length $\ell(\gamma) \leq C d(x, y)$, we have

$$
\operatorname{Mod}_{p}(\Gamma)>0
$$


It is easy to verify that a $p$-thick quasiconvex complete metric measure space is quasiconvex, that is, there is a constant $C \geq 1$ such that whenever $x, y \in X$, there is a path $\gamma$ connecting $x$ to $y$ with length $\ell(\gamma) \leq C d(x, y)$.

It was demonstrated in [DJS] that $\infty$-Poincaré inequality is equivalent to $\infty$-thick quasiconvexity. It was also shown in [DSW] that a $p$-Poincare inequality implies $p$-thick quasiconvexity, but that $p$-thick quasiconvexity does not in turn imply $p$ Poincaré inequality when $1 \leq p<\infty$.

In the following we will recall some definitions and results in [FPW] that are the starting point and motivation for our work. In the setting of spaces of homogeneous type the authors in [FPW] establish, under the following mild geometric conditions on the functional $a_{f}$, that the left-hand side of the inequality (1) can be self-improved. They consider families $\mathcal{F}$ of functions, that together with the functional $f \mapsto a_{f}$ satisfy the following natural properties:

(1) $f+\lambda, \lambda f \in \mathcal{F}$ with $a_{f}=a_{f+\lambda}$ whenever $f \in \mathcal{F}$ and $\lambda \in \mathbf{R}$,

(2) $|f| \in \mathcal{F}$ and $a_{|f|} \leq a_{f}$ if $f \in \mathcal{F}$,

(3) $f_{\lambda}:=\max \{\lambda, \min \{2 \lambda, f\}\}-\lambda \in \mathcal{F}$ whenever $f \in \mathcal{F}$ with $f \geq 0$ and $\lambda \geq 0$.

Definition 2.5. [FPW, Section 3] Let $1 \leq p<\infty$. The functional $f \mapsto a_{f}$ satisfies Condition $\left(D_{p}\right)$ if there is a constant $C>0$ such that for every $f \in \mathcal{F}$, every ball $B \in \mathcal{B}$, and every family $\left\{B_{i}\right\}$ of pairwise disjoint subballs of $B$,

$$
\sum_{i} a_{f}\left(B_{i}\right)^{p} \mu\left(B_{i}\right) \leq C a_{f}(B)^{p} \mu(B)
$$

The functional $f \mapsto a_{f}$ satisfies a truncation property if there exists a constant $C$ such that for every $f \in \mathcal{F}$ with $f \geq 0$ and $\lambda>0$, the truncations $f_{2^{k} \lambda}$ together satisfy

$$
\sum_{k \in \mathbf{Z}} a_{f_{2^{k}}}(B)^{p} \leq C a_{f}(B)^{p}
$$

for all $B \in \mathcal{B}$.

Note that by Hölder's inequality, Condition $\left(D_{p}\right)$ implies Condition $\left(D_{q}\right)$ for each $1 \leq q \leq p$.

The conditions given in the above definition, by themselves, do not indicate a first order calculus but are more associated with the $L^{p}$-theory, as we will show next; hence further conditions are needed in order to obtain information relevant to first order calculus and geometry of metric spaces.

To see that the conditions given in the above definition are associated with $L^{p_{-}}$ theory, observe that $\mathcal{F}=L^{p}(X)$ together with the functional given by

$$
a_{f}(B)=\left(f_{B} f_{B}|f(x)-f(y)|^{p} d \mu(y) d \mu(x)\right)^{1 / p}
$$

satisfies all of the conditions of [FPW]. If $f \geq 0$ and $\lambda>0$, then $f=\sum_{k \in \mathbb{Z}} f_{2^{k} \lambda}$, and moreover, for $x, y \in X$ we also have that if $f(x) \leq f(y)$ then $f_{2^{k} \lambda}(x) \leq f_{2^{k} \lambda}(y)$, and SO

$$
|f(x)-f(y)|=\sum_{k \in \mathbb{Z}}\left|f_{2^{k} \lambda}(x)-f_{2^{k} \lambda}(y)\right|
$$


Hence $a_{f}$ satisfies the truncation property with constant $C=1$. Observe that the functional given by

$$
\widetilde{a}_{f}(B)=\left(f_{B}\left|f-f_{B}\right|^{p} d \mu\right)^{1 / p}
$$

is comparable to the above functional $a_{f}$ in the sense that

$$
\begin{aligned}
\left(f_{B}\left|f-f_{B}\right|^{p} d \mu\right)^{1 / p} & \leq\left(f_{B} f_{B}|f(x)-f(y)|^{p} d \mu(y) d \mu(x)\right)^{1 / p} \\
& \leq 2\left(f_{B}\left|f-f_{B}\right|^{p} d \mu\right)^{1 / p} .
\end{aligned}
$$

Hence $\widetilde{a}_{f}$ also satisfies all the properties of [FPW] with constant $C=2$. Therefore, without any additional conditions on the functional $f \mapsto a_{f}$, any self-improvement properties of the generalized Poincare inequality are related to the $L^{p}$-theory, and is associated with the self-improvement of the left-hand side term of the inequality; this is the content of the theorem of Franchi, Perez, and Wheeden.

Theorem 2.6. [FPW, Theorem 3.1] Let $(X, d, \mu)$ be a metric measure space with $\mu$ a doubling measure and $1 \leq p<\infty$. Suppose that the functional $a_{f}$ satisfies inequality (1) for each $f \in \mathcal{F}$ and satisfies the conditions of Definition 2.5. Then there is a positive number $\eta>0$ such that for each such $f$ we have

$$
\left(f_{B}\left|f-f_{B}\right|^{p} d \mu\right)^{1 / p} \leq C a_{f}(\eta B)
$$

for all $B \in \mathcal{B}$, with $C$ independent of $B$ and $f$.

As mentioned above, the functional given in (5) satisfies the $\left(D_{p}\right)$ Condition (3) of Definition 2.5 for every $f \in L_{\mathrm{loc}}^{p}(X)$, and so by itself should not be considered to be associated with Sobolev space theory; further conditions are needed on the functional $a_{f}$ for $f \in N^{1, \infty}(X)$. In the next section we will consider two possible such conditions.

\section{First order Poincaré inequality}

In this section we will give a definition of first order Poincaré inequality as a further generalization of the $p$-Poincaré inequality described in the previous section, and consider certain conditions on this generalization that together relate the inequality to first-order calculus. We use notation similar to that adopted in [FPW]. We will consider either $\mathcal{F}=N^{1, \infty}(X)$ or $\mathcal{F}=\operatorname{LIP}^{\infty}(X)$, the collection of all bounded Lipschitz functions on $X$.

Definition 3.1. Let $\mathcal{B}$ be the collection of all balls in $X$. We say that $(X, d, \mu)$ supports a first order Poincaré inequality for $\mathcal{F}$ if for each function $f \in \mathcal{F}$ there exists a functional $a_{f}: \mathcal{B} \rightarrow[0, \infty)$ such that

$$
f_{B}\left|f-f_{B}\right| d \mu \leq a_{f}(B)
$$

for each ball $B \in \mathcal{B}$.

Our aim in this section is to establish that, under mild geometric conditions on the functional $a_{f}$, the inequality (6) encodes geometric information of the metric measure space. 
Definition 3.2. In the following, we state certain geometric conditions that might be satisfied by the functional $f \mapsto a_{f}$ in the case $\mathcal{F}=N^{1, \infty}(X)$ or $\mathcal{F}=$ $\operatorname{LIP}^{\infty}(X)$ :

(i) $a_{k f}=k a_{f}$ whenever $k \geq 0$ (scaling property).

(ii) There is a real number $\lambda \geq 1$ such that if $f_{1}, f_{2} \in \mathcal{F}$ with $f_{1}=f_{2}$ on $\lambda B$, then $a_{f_{1}}(B)=a_{f_{2}}(B)$ (locality).

(iii) (Modulus of continuity) If $\mathcal{F}=N^{1, \infty}(X)$, there exists $C>0$ such that whenever $f \in \mathcal{F}$ and $g_{f}$ is an upper gradient of $f$ such that $\left\|g_{f}\right\|_{L^{\infty}(X)} \leq 1$, then $a_{f}(B) \leq C \operatorname{rad}(B)$ for all $B \in \mathcal{B}$. If $\mathcal{F}=\operatorname{LIP}^{\infty}(X)$, the class of all bounded Lipschitz functions on $X$, then there is a constant $C>0$ such that whenever $f \in \mathcal{F}$ and $\|\operatorname{Lip} f\|_{L^{\infty}(X)} \leq 1$, then $a_{f}(B) \leq C \operatorname{rad}(B)$ for all $B \in \mathcal{B}$.

$\left(V_{p}\right)$ There exist $1 \leq p<\infty$ and $C>0$ such that whenever $f \in N^{1, \infty}(X), B \in \mathcal{B}$, and $\left\{B_{i}\right\}_{i \in \mathbf{N}}$ is a family of balls with

(a) $B_{i} \cap B_{j}=\emptyset$ for each $i \neq j$, and

(b) $\mu\left(B \backslash \bigcup_{i \in \mathbf{N}} B_{i}\right)=0$,

then

$$
\left(\frac{a_{f}(B)}{\operatorname{rad}(B)}\right)^{p} \mu(B) \leq C \sum_{i \in \mathbf{N}}\left(\frac{a_{f}\left(B_{i}\right)}{\operatorname{rad}\left(B_{i}\right)}\right)^{p} \mu\left(B_{i}\right) .
$$

In (iii), for Lipschitz functions $f$ on $X$, the local Lipschitz constant function Lip $f$ is defined by

$$
\operatorname{Lip} f(x)=\limsup _{\substack{y \rightarrow x \\ y \neq x}} \frac{|f(x)-f(y)|}{d(x, y)} .
$$

The scaling property in turn implies that if $f$ is identically zero on $X$ then $a_{f}(B)=0$ for all $B \in \mathcal{B}$. This, together with the locality property, indicates a strong locality property of $f \mapsto a_{f}$, namely, if $f$ vanishes on a ball $\lambda B$ then $a_{f}(B)=0$.

The conditions given in Definition 3.2 are intended to enforce that the functionals $a_{f}$ have the natural properties one expects of first-order calculus. The paper [HKT] considers the special case where the functional $a_{f}$ is given by a Radon measure $\nu_{f}$ :

$$
a_{f}(B)=\operatorname{rad}(B)\left(\frac{\nu_{f}(B)}{\mu(B)}\right)^{1 / p} .
$$

As with Condition (iii) of Definition 3.2, the above choice of $a_{f}(B)$ incorporates the radius of $B$. In this setting [HKT] shows that a function $f \in L^{p}(X)$ for which $a_{f}$ has an associated measure $\nu_{f}$ as above is necessarily in the Sobolev class $N^{1, p}(X)$ if $p>1$, and is in the class $B V(X)$ if $p=1$. In this paper we assume the existence of a functional $f \mapsto a_{f}$, without knowing whether such $a_{f}$ is given in terms of the integral of a function or in terms of a measure.

Theorem 3.3. [HKT, Theorem 1.1] If a function $f \in L_{\text {loc }}^{1}(X)$ has associated with it a measure $\nu_{f}$ as above with $p=1$, then $f$ is a $B V$ function on $X$ with

$$
\|D f\|(X) \leq C \nu_{f}(X) .
$$

If instead $1<p<\infty$ and $f \in L^{p}(X)$, then $f \in N^{1, p}(X)$ with

$$
\|f\|_{N^{1, p}(X)} \leq\|f\|_{L^{p}(X)}+C\left(\nu_{f}(X)\right)^{1 / p} .
$$

However, $[\mathrm{HKT}]$ does not require $\nu_{f}$ to satisfy the conditions we study, and the situation considered there is too specific on the type of functional $f \mapsto a_{f}$ to provide 
any geometric information about $X$. They also do not expect all functions in a Sobolev class to satisfy such an inequality. Furthermore, the functionals $a_{f}$ considered in [HKT] need not be driven solely by the Sobolev energy of $f$, as demonstrated by the example [HKT, Example 6.1]. The focus of this paper is different from that of [HKT]. In [HKT] the functional $a_{f}$ is of specific type, namely, it is associated with a Radon measure $\nu_{f}$, but no property is assumed on the function $f$. We, on the other hand, do not assume that $a_{f}$ is associated with a Radon measure, but we only focus on functions $f$ that are known to be in the Newtonian class $N^{1, \infty}(X)$ or is both bounded and Lipschitz continuous. We use the term first order Poincaré inequality rather than the term generalized Poincaré inequality since this latter term has been used in a much more general context (as explained above) in the papers [HKT] and [FPW].

Example 3.4. The conditions we consider in this paper are modeled after the following functional $a_{f}$. Suppose that $X$ supports a $p$-Poincaré inequality. Then, given $f \in N^{1, \infty}(X)$ and $1 \leq p<\infty$, consider the functional $a_{f}$ given by

$$
a_{f}(B)=C \operatorname{rad}(B) \inf _{g}\left(f_{\lambda B} g^{p} d \mu\right)^{1 / p}
$$

where the infimum is taken over all upper gradients $g$ of $f$ on $X$. Here $C$ and $\lambda$ are the constants related to the $p$-Poincare inequality. This functional satisfies all of the conditions of Definition 3.2, but not all metric measure spaces support a generalized Poincaré inequality with respect to this functional. The choice $a_{f} \equiv$ $\|f\|_{B M O}$ corresponds to functions in the $\operatorname{BMO}(X)$ class, but this functional in general would not satisfy any (except (i)) of the conditions given in Definition 3.2. The choice (5) of

$$
a_{f}(B)=\left(f_{B} f_{B}|f(y)-f(x)|^{p} d \mu(y) d \mu(x)\right)^{1 / p}
$$

satisfies all but Conditions (iii) and $\left(V_{p}\right)$ of Definition 3.2. If $\mathcal{F}=\operatorname{LIP}^{\infty}(X)$, then this functional satisfies Condition (iii) as well. Furthermore, if $\mathcal{F}=N^{1, \infty}(X)$ and $X$ is an $\infty$-thick quasiconvex space in the sense of [DJS], then the above choice of $f \mapsto a_{f}$ also satisfies the corresponding Condition (iii). Such thick quasiconvexity property is guaranteed if $X$ supports an $\infty$-Poincaré inequality in the sense of Section 2 . Moreover, even without knowing whether $X$ is thick quasiconvex, this choice of $a_{f}$ also can be seen to satisfy all the conditions of Definition 2.5; see the discussion in Section 2. Thus from the point of view of potential theory and Sobolev spaces, all the conditions given in Definition 3.2 seem to be needed.

Lemma 3.5. Condition $\left(V_{p}\right)$ implies Condition $\left(V_{q}\right)$ for each $q \geq p$.

Proof. Fix $f \in \mathcal{F}$ and the related functional $a_{f}$. With $B$ a ball in $X$ and $\left\{B_{i}\right\}_{i}$ a cover (up to a set of measure zero) of $B$ as in Condition $\left(V_{p}\right)$, we define the function

$$
\varphi(x)= \begin{cases}\frac{a_{f}\left(B_{i}\right)}{\operatorname{rad}\left(B_{i}\right)} & \text { if } x \in B_{i}, \\ 0 & \text { if } x \notin \bigcup_{i} B_{i} .\end{cases}
$$

Observe that

$$
\int_{B} \varphi^{p} d \mu=\sum_{i \in \mathbf{N}}\left(\frac{a_{f}\left(B_{i}\right)}{\operatorname{rad}\left(B_{i}\right)}\right)^{p} \mu\left(B_{i}\right)
$$


and so verification of Condition $\left(V_{p}\right)$ is equivalent to the verification that there exists a constant $C>0$ satisfying

$$
\left(\frac{a_{f}(B)}{\operatorname{rad}(B)}\right)^{p} \mu(B) \leq C \int_{B} \varphi^{p} d \mu
$$

By Hölder's inequality it is clear that for each $q \geq p$,

$$
\left(f_{B} \varphi^{p} d \mu\right)^{1 / p} \leq\left(f_{B} \varphi^{q} d \mu\right)^{1 / q}
$$

In particular,

$$
\left(\int_{B} \varphi^{p} d \mu\right)^{1 / p} \leq \frac{1}{(\mu(B))^{\frac{1}{q}-\frac{1}{p}}}\left(\int_{B} \varphi^{q} d \mu\right)^{1 / q},
$$

and using (7) one obtains that

$$
\left(\frac{a_{f}(B)}{\operatorname{rad}(B)}\right)^{p} \mu(B) \leq C \int_{B} \varphi^{p} d \mu \leq C(\mu(B))^{1-\frac{p}{q}}\left(\int_{B} \varphi^{q} d \mu\right)^{p / q} .
$$

Therefore,

and so

$$
\frac{a_{f}(B)}{\operatorname{rad}(B)}(\mu(B))^{1 / q} \leq C\left(\int_{B} \varphi^{q} d \mu\right)^{1 / q}
$$

$$
\left(\frac{a_{f}(B)}{\operatorname{rad}(B)}\right)^{q} \mu(B) \leq C \int_{B} \varphi^{q} d \mu .
$$

We next study some geometric properties of metric measure spaces that satisfy some of the conditions in Definition 3.2.

One of the most useful geometric consequences of the combination of the properties of completeness, doubling of the measure, and the support of a $p$-Poincare inequality is the quasiconvexity of a metric space that has all these properties: there exists a constant such that each pair of points can be connected with a curve whose length is at most the constant times the distance between the points (see [Se]). In what follows we will see that the first order Poincaré inequality that satisfies one of the conditions of Definition 3.2 also encodes this type of geometric information. As pointed out in [HKT, Example 6.1], all the conditions except (iii) of Definition 3.2 together will not imply that the metric measure space $X$ is even connected, let alone quasiconvex.

Theorem 3.6. Let $(X, d, \mu)$ be a complete metric space with $\mu$ doubling. Assume also that $(X, d, \mu)$ supports a first order Poincare inequality with either $\mathcal{F}=$ $N^{1, \infty}(X)$ or $\mathcal{F}=\operatorname{LIP}^{\infty}(X)$, and the functional $f \mapsto a_{f}$ satisfies the geometric Condition (iii) of Definition 3.2. Then $(X, d, \mu)$ is quasiconvex.

Proof. Let $\varepsilon>0$. We say that $x, z \in X$ lie in the same $\varepsilon$-component of $X$ if there exists an $\varepsilon$-chain joining $x$ with $z$, that is, there exists a finite sequence $z_{0}, z_{1}, \ldots, z_{n}$ such that $z_{0}=x, z_{n}=z$ and $d\left(z_{i}, z_{i+1}\right) \leq \varepsilon$ for all $i=0, \ldots, n-1$. Note that $\varepsilon$-components are open sets, and the distance between two distinct components is at least $\varepsilon$. Let $U$ be such a component. We wish to show that $U=X$.

If $x$ and $y$ lie in different $\varepsilon$-components, say $U$ and $V$, then it is obvious that there does not exist a rectifiable path joining $x$ and $y$. Thus, the function $g \equiv 0$ is an upper gradient for $f_{n}=n \cdot \chi_{U}$ (observe that because such components are open sets, it follows also that $\operatorname{Lip} f_{n} \equiv 0$; this observation is relevant for the case 
that $\left.\mathcal{F}=\operatorname{LIP}^{\infty}(X)\right)$. Obviously, $f_{n} \in N^{1, \infty}(X)$ is $n / \varepsilon$-Lipschitz continuous, with $\|g\|_{L^{\infty}(X)} \leq 1$ and $\left\|\operatorname{Lip} f_{n}\right\|_{L^{\infty}(X)} \leq 1$. Therefore by choosing a ball $B$ such that both $B \cap U \neq \emptyset$ and $B \backslash U \neq \emptyset$, and applying first order Poincaré inequality and Condition (iii), we obtain

$$
\begin{aligned}
0 & <\frac{\mu(B \cap U) \mu(B \backslash U)}{\mu(B)^{2}} \leq \frac{2 n \mu(B \cap U) \mu(B \backslash U)}{\mu(B)^{2}}=f_{B}\left|f_{n}-\left(f_{n}\right)_{B}\right| d \mu \\
& \leq a_{f_{n}}(B) \leq C \operatorname{rad}(B)<\infty
\end{aligned}
$$

for each $n \in \mathbf{N}$, which is not possible. Therefore, all the points of $X$ lie in the same $\varepsilon$-component.

Now, let us fix $x, y \in X$ and prove that there exists a path $\gamma$ joining $x$ and $y$ such that $\ell(\gamma) \leq C d(x, y)$, where $C$ is a constant which depends only on the doubling constant and the constants involved in the first order Poincaré inequality. We define the $\varepsilon$-distance of $x$ to $z$ to be

$$
\rho_{x, \varepsilon}(z):=\inf \sum_{i=0}^{N-1} d\left(z_{i}, z_{i+1}\right),
$$

where the infimum is taken over all finite $\varepsilon$-chains $\left\{z_{i}\right\}$ from $x$ to $z$. Note that $\rho_{x, \varepsilon}(z)<\infty$ for all $z \in X$. In addition, if $d(z, w) \leq \varepsilon$ then $\left|\rho_{x, \varepsilon}(z)-\rho_{x, \varepsilon}(w)\right| \leq d(z, w)$. Hence, the function $\widehat{\rho_{x, \varepsilon}}$ given by

$$
\widehat{\rho_{x, \varepsilon}}(z)=\min \left\{\rho_{x, \varepsilon}(z), \varepsilon^{-1}\right\}
$$

is a locally 1-Lipschitz function, in particular, every point is a Lebesgue point of $\widehat{\rho_{x, \varepsilon}}$ and in addition, for all $\varepsilon>0$, the function $g \equiv 1$ is an upper gradient of $\widehat{\rho_{x, \varepsilon}} \in N^{1, \infty}(X)$. To take care of the situation $\mathcal{F}=\operatorname{LIP}^{\infty}(X)$, we now show that $\widehat{\rho_{x, \varepsilon}}$ is Lipschitz continuous. Let $y, z \in X$. If $d(y, z)<\varepsilon$, then clearly $\left|\widehat{\rho_{x, \varepsilon}}(y)-\widehat{\rho_{x, \varepsilon}}(z)\right| \leq$ $d(y, z)$. If $d(y, z) \geq \varepsilon$, then we have an $\varepsilon$-chain from $y$ to $z$. Let $N$ be the number of points in this chain. Then using the fact that $\widehat{\rho_{x, \varepsilon}}$ is bounded above by $1 / \varepsilon$ and the fact that $d(y, z) \geq \varepsilon$,

$$
\left|\widehat{\rho_{x, \varepsilon}}(y)-\widehat{\rho_{x, \varepsilon}}(z)\right| \leq\left|\widehat{\rho_{x, \varepsilon}}(y)\right|+\left|\widehat{\rho_{x, \varepsilon}}(z)\right| \leq \frac{2}{\varepsilon}=\frac{2}{\varepsilon d(y, z)} d(y, z) \leq \frac{2}{\varepsilon^{2}} d(y, z) .
$$

It follows that $\widehat{\rho_{x, \varepsilon}}$ is $\max \left\{1,2 / \varepsilon^{2}\right\}$-Lipschitz on $X$.

For each $i \in \mathbf{Z}$, define $B_{i}=B\left(x, 2^{1-i} d(x, y)\right)$ if $i \geq 0$, and $B_{i}=B\left(y, 2^{1+i} d(x, y)\right)$ if $i \leq-1$. Thus, a telescopic argument, together with first order Poincaré inequality and Condition (iii), gives us the following chain of inequalities:

$$
\begin{aligned}
\left|\widehat{\rho_{x, \varepsilon}}(y)\right|=\left|\widehat{\rho_{x, \varepsilon}}(x)-\widehat{\rho_{x, \varepsilon}}(y)\right| & \leq \sum_{i \in \mathbf{Z}}\left|\int_{B_{i}} \widehat{\rho_{x, \varepsilon}} d \mu-f_{B_{i+1}} \widehat{\rho_{x, \varepsilon}} d \mu\right| \\
& \leq C \sum_{i \in \mathbf{Z}} f_{B_{i}}\left|\widehat{\rho_{x, \varepsilon}}-f_{B_{i}} \widehat{\rho_{x, \varepsilon}} d \mu\right| d \mu \\
& \leq C \sum_{i \in \mathbf{Z}} a_{\widehat{\rho_{x, \varepsilon}}}\left(B_{i}\right) \leq C \sum_{i \in \mathbf{Z}} C d(x, y) 2^{-|i|} \leq C d(x, y),
\end{aligned}
$$

where $C$ is a constant that depends only on $X$. For fixed $x, y \in X$ observe that for sufficiently small $\varepsilon>0$ we have $\widehat{\rho_{x, \varepsilon}}(y)=\rho_{x, \varepsilon}(y)$.

Since $X$ is complete, the existence of a non trivial doubling measure implies that closed balls are compact. Using a standard limiting argument, which involves 
Arzela-Ascoli's theorem and inequality (8), we can construct a 1-Lipschitz rectifiable path connecting $x$ and $y$ with length at most $C d(x, y)$. For further details about the construction of the curve we refer the reader to [Ko, Theorem 3.1].

In the following we try to weaken Condition (iii). A metric space is said to be $\varphi$-convex if there exists a homeomorphism $\varphi:[0, \infty) \rightarrow[0, \infty)$ such that each pair of distinct points $x$ and $y$ can be joined by a path whose length does not exceed $\varphi(d(x, y))$. A $C$-quasiconvex space is $\varphi$-convex with $\varphi(t)=C t$.

Definition 3.7. We say that the functional $f \mapsto a_{f}$ satisfies Condition (iiia) if there exist two homeomorphisms $\varphi, \psi:[0, \infty) \rightarrow[0, \infty)$ satisfying the following two conditions:

(I) There is a constant $C>0$ such that whenever $f \in N^{1, \infty}(X)$ has an upper gradient $g_{f}$ with $\left\|g_{f}\right\|_{L^{\infty}(X)} \leq 1$, we must have $a_{f}(B) \leq C \psi(\operatorname{rad}(B))$ for each $B \in \mathcal{B}$.

(II) $\sum_{i \in \mathbf{N}} \psi\left(2^{-i} r\right) \leq \varphi(r)$ for $r \in[0, \infty)$.

Observe that Condition (iii) of Definition 3.2 implies Condition (iiia). This is seen by making the choice of $\psi(t)=2 t=\varphi(t)$.

We point out that we assume in this paper that $X$ is a complete metric space with $\mu$ a doubling measure.

Lemma 3.8. Let $X$ be locally rectifiably connected. Assume that $(X, d, \mu)$ supports a first order Poincaré inequality for $\mathcal{F}=N^{1, \infty}(X)$ with the functional $f \mapsto a_{f}$ satisfying Condition (iiia). Then $(X, d, \mu)$ is $\varphi$-convex.

We do not at this time know whether the above lemma holds if $\mathcal{F}=\operatorname{LIP}^{\infty}(X)$, even though for Theorem 3.6 this choice also is valid. The reason is that the proof of Theorem 3.6 is not adaptable to the proof of this lemma. Indeed, the function constructed in the proof of Theorem 3.6 can also be considered here, but all that leads to is the estimate that for each $x \in X$ and $\varepsilon>0, \widehat{\rho_{\varepsilon, x}}(y) \leq C \psi(d(x, y))$. Unlike with quasiconvex curves, this does not lead to the existence of rectifiable curves connecting $x$ to $y$ with length at most $C \psi(d(x, y))$; hence the difficulty.

Proof. Fix $x \in X$ and set $U_{x}$ to be the rectifiable path-component of $X$ containing $x$. Since $X$ is locally rectifiably connected, it follows that $U_{x}$ is open; on the other hand, $X \backslash U_{x}$ is also open for the same reason. Thus by considering the function $f$ given by $f(y)=\inf _{\gamma} \ell(\gamma)$ with the infimum taken over all rectifiable paths in $X$ connecting $x$ to $y$ when $y \in U_{x}$, and $f(y)=0$ if $y \in X \backslash U_{x}$, we see from a repeat of the argument found in the proof of Theorem 3.6 that $X=U_{x}$.

Now we fix $x \in X$ and $\varepsilon>0$, and set

$$
\rho_{x, \varepsilon}(y)=\min \left\{\inf _{\gamma} \ell(\gamma), \varepsilon^{-1}\right\}
$$

where the infimum is taken over all rectifiable paths $\gamma$ connecting $\bar{B}(x, \varepsilon)$ to $y$. Note that $\rho_{x, \varepsilon}=0$ on $B(x, \varepsilon)$, and that $g \equiv 1$ is an upper gradient of $\rho_{x, \varepsilon}$. It follows from the results in [JJRRS] (see also [HKST]) that $\rho_{x, \varepsilon}$ is measurable, and so belongs to $N^{1, \infty}(X)$. Fix $y \in X \backslash B(x, 2 \varepsilon)$ such that $y$ is a Lebesgue point of $\rho_{x, \varepsilon}$.

For each $i \in \mathbf{Z}$, define $B_{i}=B\left(x, 2^{1-i} d(x, y)\right)$ if $i \geq 0$, and $B_{i}=B\left(y, 2^{1+i} d(x, y)\right)$ if $i \leq-1$. Thus, a telescopic argument, together with first order Poincaré inequality 
and Condition (iiia), gives us the following chain of inequalities:

$$
\begin{aligned}
\left|\rho_{x}(y)\right|=\left|\rho_{x, \varepsilon}(x)-\rho_{x, \varepsilon}(y)\right| & \leq \sum_{i \in \mathbf{Z}}\left|f_{B_{i}} \rho_{x, \varepsilon} d \mu-f_{B_{i+1}} \rho_{x, \varepsilon} d \mu\right| \\
& \leq C \sum_{i \in \mathbf{Z}} f_{B_{i}}\left|\rho_{x, \varepsilon}-f_{B_{i}} \rho_{x, \varepsilon} d \mu\right| d \mu \\
& \leq C \sum_{i \in \mathbf{Z}} a_{\rho_{x}, \varepsilon}\left(B_{i}\right) \leq C \sum_{i \in \mathbf{Z}} \psi\left(d(x, y) 2^{-|i|}\right) \leq 3 C \varphi(d(x, y))
\end{aligned}
$$

where $C$ is a constant that depends only on $X$. Thus there is a set $Z_{\varepsilon} \subset X$ with $\mu\left(Z_{\varepsilon}\right)=0$ such that every $y \in X \backslash Z_{\varepsilon} \cup B(x, 2 \varepsilon)$ can be connected to $\bar{B}(x, \varepsilon)$ by a path of length at most $C \varphi(d(x, y))$ or else each path connecting $y$ to $\bar{B}(x, \varepsilon)$ has length at least $\varepsilon^{-1}$. Let $Z:=\bigcup_{n \in N} Z_{1 / n}$; note that $\mu(Z)=0$. Fix $y \in X \backslash Z$ with $y \neq x$. Then for each $n \in \mathbf{N}$ either each path connecting $\bar{B}(x, \varepsilon)$ to $y$ has length at least $n$, or else we can find a path $\gamma_{n}$ connecting $y$ to a point $x_{n} \in X$ with $d\left(x, x_{n}\right) \leq 1 / n$ such that $\ell\left(\gamma_{n}\right) \leq C \varphi(x, y)$. Since $X$ is complete, the existence of a non trivial doubling measure implies that closed balls are compact. Using a standard limiting argument involving Arzela-Ascoli's theorem, and the fact that $X$ is rectifiably connected (see the first part of the proof), we can construct a 1-Lipschitz rectifiable path connecting $x$ and $y$ as in the proof of [Ko, Theorem 3.1], with length at most $C \varphi(d(x, y))$.

Finally, another application of Arzela-Ascoli's theorem together with the fact that $X \backslash Z$ is dense in $X$, allows us to connect each $y \in X$ to $x$ by a rectifiable path of length at most $C \varphi(d(x, y))$. Since the above argument holds for all $x \in X$, the lemma is now proved.

Notice that the space in Example 3.9 below is a $\varphi$-convex space which is not quasiconvex.

Example 3.9. Let us consider the following domain $X$ given by

$$
X:=\left\{\left(x_{1}, x_{2}\right) \in \mathbf{R}^{2}:-1 \leq x_{1} \leq 1 \text { and }-x_{1}^{2} \leq x_{2}^{3} \leq x_{1}^{2}\right\},
$$

endowed with the restriction to $X$ of the Euclidean metric of $\mathbf{R}^{2}$, denoted $d$, and the 2-dimensional Lebesgue measure $\mu=\mathscr{L}_{\mid X}^{2}$. Observe that $(X, d, \mu)$ is a complete and doubling non-quasiconvex metric space. Therefore, it does not support any $p$-Poincaré inequality, $1 \leq p \leq \infty$. However, we will see that it supports a first order Poincare inequality. First of all notice that each function $f \in N^{1, \infty}(X)$ is $\alpha$-Hölder continuous with $\alpha=2 / 3$. Indeed, if $x, y \in X, 0<\varepsilon<d(x, y) / 10$, and $E \subset B(x, \varepsilon)$ and $F \subset B(y, \varepsilon)$ are measurable sets such that $\mu(E) \mu(F)>0$, then the $\infty$-modulus of the collection of paths in $X$ connecting $E$ to $F$ with length no more than $10 d(x, y)^{\alpha}$ is positive. To see this, note that if both $x$ and $y$ belong to the same lobe of $X$ (that is, with $x=\left(x_{1}, x_{2}\right)$ and $y=\left(y_{1}, y_{2}\right)$, both $x_{1}$ and $y_{1}$ have the same sign), then a standard Fubini-type argument applied to the tube of straight line segments connecting $F$ to $E$ shows that the $p$-modulus of the sub-family of straight line segments is positive for any $1 \leq p<\infty$, and hence so is the $\infty$-modulus. If $x$ and $y$ belong to different lobes of $X$, then the sub-family of curves formed by connecting $F$ to the point $(0,0) \in \mathbf{R}^{2}$ by straight line segments and then from $(0,0)$ to $E$, has positive $p$-modulus when $p>2$, see [Va]. These curves have length no more than $10 d(x, y)^{\alpha}$. 
From the above observation, together with the arguments found in the proof of [DJS, Lemma 4.4], we see that functions in $N^{1, \infty}(X)$ are 2/3-Hölder continuous. In particular, given a ball $B \in \mathcal{B}$ and $x, y \in B$,

$$
|f(x)-f(y)| \leq C d(x, y)^{\alpha}\left\|g_{f}\right\|_{L^{\infty}(C B)},
$$

whenever $g_{f}$ is an upper gradient of $f$. If we define the functional $a_{f}: \mathcal{B} \rightarrow[0, \infty)$ by $a_{f}(B)=C \operatorname{rad}(B)^{\alpha}\left\|g_{f}\right\|_{L^{\infty}(C B)}$ we have that

$$
\begin{aligned}
& f_{B}\left|f-f_{B}\right| d \mu \leq f f_{B}|f(y)-f(x)| d \mu(y) d \mu(x) \\
& \leq C \int_{B} f_{B} d(x, y)^{\alpha}\left\|g_{f}\right\|_{L^{\infty}(C B)} d \mu(y) d \mu(x) \\
& \leq C \operatorname{rad}(B)^{\alpha}\left\|g_{f}\right\|_{L^{\infty}(C B)}=a_{f}(B)
\end{aligned}
$$

for each ball $B \in \mathcal{B}$, as wanted. That is, with the choices of $\psi(r)=r^{2 / 3}$ and $\varphi(r)=\left(\sum_{i \in \mathbf{N}} 2^{-2 i / 3}\right) r^{2 / 3}$, we know that Condition (iiia) holds. However, the stronger Condition (iii) fails, for $X$ is not quasiconvex.

The book of Saloff-Coste [S-C], Section 5.6.3, has a nice discussion of the Poincaré type inequalities for Riemannian manifolds of negative lower-bounded Ricci curvature, and in this case the function $\psi(r)=C r e^{C_{n} \sqrt{-K} r}$. Here $K$ is the lower bound for the Ricci curvature, $K>0$, and $C_{n}$ depends on the dimension $n$ of the manifold. In this case note that there are such manifolds that are geodesic spaces (for example, the model spaces $\mathbb{H}^{n}$ ), and so the result of Lemma 3.8 is far from being optimal. Observe that we can choose $\varphi=\psi$ for such $\psi$ in this lemma. See [BJM], [CW], [FW] for discussions on other choices of $\psi$.

\section{First order Poincaré vs. $p$-Poincaré inequalities}

In this section we state the main theorem of this work. The following theorem relates first order Poincaré inequalities to $p$-Poincaré inequalities when the functional satisfies the conditions of Definition 3.2.

Theorem 4.1. If $(X, d, \mu)$ is a complete metric space with $\mu$ a doubling measure, then it supports a first order Poincaré inequality for $\mathcal{F}=N^{1, \infty}(X)$ or $\mathcal{F}=\operatorname{LIP}^{\infty}(X)$, with the functional $f \mapsto a_{f}$ satisfying the geometric Conditions (i), (ii), (iii) and $\left(V_{p}\right)$ of Definition 3.2, if and only if $(X, d, \mu)$ supports a p-Poincaré inequality.

Proof. First assume that $(X, d, \mu)$ supports a $p$-Poincaré inequality. Then, there are constants $\lambda_{p}, C_{p}>0$ such that when $f: X \rightarrow \mathbf{R} \cup\{-\infty, \infty\}$ is a measurable function and $g: X \rightarrow[0, \infty]$ is an upper gradient of $f$, and $B$ is a ball in $X$,

$$
f_{B}\left|f-f_{B}\right| d \mu \leq C_{p} \operatorname{rad}(B)\left(f_{\lambda_{p} B} g^{p} d \mu\right)^{1 / p}
$$

We know then that $X$ is quasiconvex. By a bi-Lipschitz change in the metric on $X$, we can assume without loss of generality that $X$ is a geodesic space. Hence by the results of [HaK], by increasing the value of $C_{p}$ we can assume that $\lambda_{p}=1$.

Note that given a function $f$ on $X$, the set of all upper gradients of $f$ that are in the class $L^{p}(X)$ is a convex subset of $L^{p}(X)$. A delicate application of Mazur's lemma shows that there is a unique non-negative Borel measurable function, denoted 
$g_{f}$, in the closure (in $L^{p}(X)$ ) of this convex set such that whenever $g \in L^{p}(X)$ is in the closure of this convex set, we have $g_{f} \leq g$ almost everywhere in $X$; see for example [Sh1] or [Ha]. The function $g_{f}$ is said to be the minimal p-weak upper gradient of $f$. Note that Equation (10) holds if we replace $g$ on the right-hand side by $g_{f}$.

Now, for each function $f \in N^{1, \infty}(X)$, choose the minimal $p$-weak upper gradient $g_{f}$ and define the functional $a_{f}: \mathcal{B} \rightarrow[0, \infty)$ as

$$
a_{f}(B)=C_{p} \operatorname{rad}(B)\left(f_{B} g_{f}^{p} d \mu\right)^{1 / p} .
$$

With this choice of $a_{f}$, Conditions (i), (ii), (iii) and $\left(V_{p}\right)$ hold. Notice that, denoting $C_{p}^{p}$ by the generic constant $C$,

$$
\left(\frac{a_{f}(B)}{\operatorname{rad}(B)}\right)^{p} \mu(B)=C \int_{B} g_{f}^{p} d \mu .
$$

To prove the converse, by [Ke3, Theorem 2] it is enough to verify inequality (2) of Definition 2.3 for compactly supported Lipschitz functions with compactly supported Lipschitz upper gradients. Recall by Theorem 3.6 that $X$ is quasiconvex.

Let $f$ be a compactly supported Lipschitz function and $g$ a continuous upper gradient of $f$. In particular, $f \in \operatorname{LIP}^{\infty}(X) \subset \mathcal{F}$. By hypothesis, there exists a functional $a_{f}: \mathcal{B} \rightarrow[0, \infty)$ supporting first order Poincaré inequality. Let $h_{f}: X \rightarrow$ $[0, \infty]$ be a function given by

$$
h_{f}(x)=\limsup _{r \rightarrow 0} \sup _{\substack{x \in B \in \mathcal{B} \\ \operatorname{rad}(B)<r}} \frac{a_{f}(B)}{\operatorname{rad}(B)}=\lim _{r \rightarrow 0} \sup _{\substack{x \in B \in \mathcal{B} \\ \operatorname{rad}(B)<r}} \frac{a_{f}(B)}{\operatorname{rad}(B)} .
$$

It is easy to see that $h_{f}$ is a Borel measurable function.

Claim. For each $x \in X$,

$$
h_{f}(x) \leq C g(x) .
$$

To prove this claim, we fix $x \in X$ and $\varepsilon>0$. Since $g$ is continuous, there exists a ball $B_{x} \ni x$ such that $g \leq g(x)+\varepsilon$ on $B_{x}$. Now let $B \in \mathcal{B}$ such that $x \in B$ and $2 C_{q} \lambda \operatorname{rad}(B)<r_{x} / 2$, where $r_{x}$ is the radius of $B_{x}$. Then $2 C_{q} \lambda B \subset B_{x}$. Here $C_{q}$ is the quasiconvexity constant of $X$ from Theorem 3.6. For $y, z \in \lambda B$ one can construct a quasiconvex path $\gamma_{y z} \in C_{q} \lambda B$ connecting $y$ and $z$ such that $\ell\left(\gamma_{y z}\right) \leq C_{q} d(y, z)$. Thus,

$$
|f(y)-f(x)| \leq \int_{\gamma_{y z}} g d s \leq(g(x)+\varepsilon) \ell\left(\gamma_{y z}\right) \leq C_{q}(g(x)+\varepsilon) d(y, z),
$$

and so $f$ is $C_{q}(g(x)+\varepsilon)$-Lipschitz continuous on the ball $\lambda B$. By the McShane extension theorem (see [He] for example), we can obtain an extension $F$ of $f$ defined on all $X$ which is $C_{q}(g(x)+\varepsilon)$-Lipschitz on $X$ and so $F$ has the constant function $k=C_{q}(g(x)+\varepsilon)$ as an upper gradient on $X$. By Condition (i) of $a_{f}$ we have that $a_{k F}=k a_{F}$ and by Condition (iii) (for either choice of $\mathcal{F}$ ) we then have that

$$
a_{F}(B) \leq C \operatorname{rad}(B) C_{q}(g(x)+\varepsilon) .
$$

Observe that for each $z \in X$ we have $\operatorname{Lip} F(z) \leq C_{q}(g(x)+\varepsilon)$, and so the above argument works also for the case that $\mathcal{F}=\operatorname{LIP}^{\infty}(X)$. 

and so

Since $f=F$ on $\lambda B$, by the locality property (ii) we have that $a_{f}(B)=a_{F}(B)$,

$$
a_{f}(B) \leq C \operatorname{rad}(B) C_{q}(g(x)+\varepsilon) .
$$

Thus we deduce that for $B \in \mathcal{B}$ such that $x \in B$ and $\operatorname{rad}(B)$ sufficiently small, $h_{f}(x) \leq C C_{q}(g(x)+\varepsilon)$. Letting $\varepsilon \rightarrow 0$ we conclude the proof of the claim.

Let $\delta>0$. Fix $B \in \mathcal{B}$. Then for each $\varepsilon>0$ and each $x \in B$, there exists $B_{x}^{\varepsilon} \in \mathcal{B}$ with $B_{x}^{\varepsilon} \subset B$ and $\operatorname{rad}\left(B_{x}^{\varepsilon}\right)<\varepsilon$ such that

$$
a_{f}\left(B_{x}^{\varepsilon}\right) \leq\left(\delta+h_{f}(x)\right) \operatorname{rad}\left(B_{x}^{\varepsilon}\right) .
$$

We now fix $\varepsilon_{0}$, and consider a cover $\left\{B_{x}^{\varepsilon}\right\}_{x \in B ; \varepsilon<\varepsilon_{0}}$ of $B$. By the Vitali covering theorem there exists a countable pairwise disjoint collection of such balls $\left\{B_{i}^{\varepsilon_{0}}\right\}_{i \in \mathbf{N}}$ such that

$$
\mu\left(B \backslash \bigcup_{i} B_{i}^{\varepsilon_{0}}\right)=0
$$

Recall that we assume $\mu$ to be doubling, so this theorem holds; see [He]. Let $x_{i}^{\varepsilon_{0}} \in B_{i}^{\varepsilon_{0}}$ be such that

$$
a_{f}\left(B_{i}^{\varepsilon_{0}}\right) \leq\left(\delta+h_{f}\left(x_{i}^{\varepsilon_{0}}\right)\right) \operatorname{rad}\left(B_{i}^{\varepsilon_{0}}\right) .
$$

By Condition $\left(V_{p}\right)$ there exists a constant $C>0$ such that

$$
\begin{aligned}
\left(\frac{a_{f}(B)}{\operatorname{rad}(B)}\right)^{p} \mu(B) & \leq C \sum_{i \in \mathbf{N}}\left(\frac{a_{f}\left(B_{i}^{\varepsilon_{0}}\right)}{\operatorname{rad}\left(B_{i}^{\varepsilon_{0}}\right)}\right)^{p} \mu\left(B_{i}^{\varepsilon_{0}}\right) \\
& \leq C \sum_{i \in \mathbf{N}}\left(\delta+h_{f}\left(x_{i}^{\varepsilon_{0}}\right)\right)^{p} \mu\left(B_{i}^{\varepsilon_{0}}\right) \\
& \leq C 2^{p} \sum_{i \in \mathbf{N}}\left(\delta^{p}+h_{f}\left(x_{i}^{\varepsilon_{0}}\right)^{p}\right) \mu\left(B_{i}^{\varepsilon_{0}}\right) \\
& \leq C 2^{p} \sum_{i \in \mathbf{N}} \delta^{p} \mu\left(B_{i}^{\varepsilon_{0}}\right)+C 2^{p} \sum_{i \in \mathbf{N}} h_{f}\left(x_{i}^{\varepsilon_{0}}\right)^{p} \mu\left(B_{i}^{\varepsilon_{0}}\right) \\
& \leq C 2^{p} \delta^{p} \mu(B)+C 2^{p} \sum_{i \in \mathbf{N}} g\left(x_{i}^{\varepsilon_{0}}\right)^{p} \mu\left(B_{i}^{\varepsilon_{0}}\right) . \quad \text { by }(12)
\end{aligned}
$$

Since $\operatorname{rad}\left(B_{x}^{\varepsilon_{0}}\right)<\varepsilon_{0}$, the mesh of the collection $\left\{B_{i}^{\varepsilon_{0}}\right\}_{i \in \mathbf{N}}$ is at most $\varepsilon_{0}$. Moreover, $g$ is continuous and so $\int_{B} g^{p} d \mu$ is computable as Riemannian sums:

$$
\lim _{\varepsilon_{0} \rightarrow 0} \sum_{i \in \mathbf{N}} g\left(x_{i}^{\varepsilon_{0}}\right)^{p} \mu\left(B_{i}^{\varepsilon_{0}}\right)=\int_{B} g^{p} d \mu .
$$

If we let $\varepsilon_{0} \rightarrow 0$,

$$
\left(\frac{a_{f}(B)}{\operatorname{rad}(B)}\right)^{p} \mu(B) \leq C 2^{p} \delta^{p} \mu(B)+C 2^{p} \int_{B} g^{p} d \mu .
$$

Now letting $\delta \rightarrow 0$ we get

$$
\left(\frac{a_{f}(B)}{\operatorname{rad}(B)}\right)^{p} \mu(B) \leq C \int_{B} g^{p} d \mu
$$

that is,

$$
a_{f}(B) \leq C^{1 / p} \operatorname{rad}(B)\left(f_{B} g^{p} d \mu\right)^{1 / p}
$$


Applying the first order Poincaré inequality we get

$$
f_{B}\left|f-f_{B}\right| d \mu \leq a_{f}(B) \leq C^{1 / p} \operatorname{rad}(B)\left(f_{B} g^{p} d \mu\right)^{1 / p} .
$$

By the self-improvement result in [KZ], together with the proof of Theorem 4.1 we see that the following corollary holds true.

Corollary 4.2. Given a functional $a_{f}$ satisfying Conditions (i), (ii), (iii), and $\left(V_{p}\right)$ of Definition 3.2 for some $p>1$, there is some $1 \leq q<p$ and a functional $\tilde{a}_{f}$ such that $\tilde{a}_{f}$ satisfies (i), (ii), (iii) and $\left(V_{q}\right)$.

Remark 4.3. Observe that in the proof of Theorem 4.1, we have obtained that

$$
a_{f}(B) \leq C \operatorname{rad}(B)\left(f_{B} g^{p} d \mu\right)^{1 / p}
$$

whenever $g$ is a continuous upper gradient of $f$. However, we do not know whether the two quantities above are comparable, nor whether the same inequality holds true when the upper gradient is not continuous (in particular, minimal $p$-weak upper gradient of $f$ ). Furthermore, we do not know in this generality whether $a_{f}$ is comparable to the integral on the right hand side of the above inequality for some weak upper gradient $g$ of $f$.

In light of the above remark, it is worth trying to understand what $a_{f}(B)$ is under some additional conditions on $a_{f}$. The proof of Theorem 4.1 shows also that

$$
a_{f}(B) \leq C \operatorname{rad}(B)\left(f_{B} h_{f}^{p} d \mu\right)
$$

provided that $\int_{B} h_{f}^{p} d \mu$ can be expressed as a limit of Riemann sums, where $h_{f}$ is given by the formula (11). Not all nonnegative functions in $L^{p}(B)$ have this property. To get around this issue, we consider $h_{r}$ for each $r>0$ as follows:

$$
h_{r}(x)=\sup _{\substack{x \in B \in \mathcal{B} \\ \operatorname{rad}(B)<r}} \frac{a_{f}(B)}{\operatorname{rad}(B)} .
$$

It is clear that $h_{r} \geq h_{f}$ and that $h_{r}$ is upper semicontinuous, and hence can be approximated in $L^{p}(X)$ by a monotonic decreasing sequence of continuous functions $h_{r, j}$. Thus we can replace $h_{f}$ in (14) by $h_{r, j}$; then letting $j \rightarrow \infty$ we see that we can replace $h_{f}$ with $h_{r}$ in (14). Now, an application of the monotone convergence theorem tells us that we are allowed to have $h_{f}$ in (14).

Assume now that in addition to the hypothesis of Theorem 4.1 we have the following reverse of Condition $\left(V_{p}\right)$ :

$r\left(V_{p}\right)$ There exists $1 \leq p<\infty$ such that whenever $f \in N^{1, \infty}(X)$ and $B \in \mathcal{B}$, if $\left\{B_{i}\right\}_{i \in \mathbf{N}}$ is a family with

(a) $B_{i} \in \mathcal{B}$ for $i \in \mathbf{N}$ with $B_{i} \subset B$,

(b) $B_{i} \cap B_{j}=\emptyset$ for each $i \neq j$, and

(c) $\mu\left(B \backslash \bigcup_{i \in \mathbf{N}} B_{i}\right)=0$,

then there exists a constant $C>0$ such that

$$
\left(\frac{a_{f}(B)}{\operatorname{rad}(B)}\right)^{p} \mu(B) \geq C \sum_{i \in \mathbf{N}}\left(\frac{a_{f}\left(B_{i}\right)}{\operatorname{rad}\left(B_{i}\right)}\right)^{p} \mu\left(B_{i}\right) .
$$


Now, following the proof of Theorem 4.1, let us define the function

$$
h_{f}^{-}(x)=\lim _{r \rightarrow 0} \inf _{\substack{x \in B \in \mathcal{B} \\ \operatorname{rad}(B)<r}} \frac{a_{f}(B)}{\operatorname{rad}(B)} .
$$

This function is $\mu$-measurable but is not so easy to compare directly with $a_{f}$, so instead we consider for each $\varepsilon>0$,

$$
h_{f, \varepsilon}^{-}(x)=\inf _{\substack{x \in B \in \mathcal{B} \\ \operatorname{rad}(B)<\varepsilon}} \frac{a_{f}(B)}{\operatorname{rad}(B)} .
$$

Then for $\varepsilon>0$ and $x \in B$, whenever $y \in B_{x}^{\varepsilon} \in \mathcal{B}$ with $\operatorname{rad}\left(B_{x}^{\varepsilon}\right)<\varepsilon$, we have

$$
h_{f, \varepsilon}^{-}(y) \leq \frac{a_{f}\left(B_{x}\right)}{\operatorname{rad}\left(B_{x}\right)} .
$$

Let us consider a cover $\left\{B_{x}^{\varepsilon}\right\}_{x \in B, \varepsilon<\varepsilon_{0}}$ of $B$ by balls $B_{x}^{\varepsilon}, x \in B$, such that $\operatorname{rad}\left(B_{x}^{\varepsilon}\right)<$ $\varepsilon$ and $B_{x}^{\varepsilon} \subset B$. By the Vitali covering theorem, there exists a countable pairwise disjoint collection of such balls $\left\{B_{i}^{\varepsilon}\right\}_{i \in \mathbf{N}}$ such that $\mu\left(B \backslash \bigcup_{i} B_{i}^{\varepsilon}\right)=0$. Now,

$$
\begin{aligned}
\left(\frac{a_{f}(B)}{\operatorname{rad}(B)}\right)^{p} \mu(B) & \geq C \sum_{i \in \mathbf{N}}\left(\frac{a_{f}\left(B_{i}^{\varepsilon}\right)}{\operatorname{rad}\left(B_{i}^{\varepsilon}\right)}\right)^{p} \mu\left(B_{i}^{\varepsilon}\right)=C \sum_{i \in \mathbf{N}} \int_{B_{i}^{\varepsilon}}\left(\frac{a_{f}\left(B_{i}^{\varepsilon}\right)}{\operatorname{rad}\left(B_{i}^{\varepsilon}\right)}\right)^{p} d \mu \\
& \geq C \sum_{i \in \mathbf{N}} \int_{B_{i}^{\varepsilon}}\left(h_{f, \varepsilon}^{-}\right)^{p} d \mu .
\end{aligned}
$$

Using the fact that $h_{f, \varepsilon}^{-}$monotonically increases to $h_{f}^{-}$, we get that

$$
a_{f}(B) \geq C^{1 / p} \operatorname{rad}(B)\left(f_{B}\left(h_{f}^{-}\right)^{p} d \mu\right)^{1 / p} .
$$

In general we do not know that $h_{f} \approx h_{f}^{-}$. However, if $a_{f}$ is given via a Radon measure $\nu_{f}$ as in [HKT], that is,

$$
a_{f}(B)=\operatorname{rad}(B)\left(\frac{\nu_{f}(B)}{\mu(B)}\right)^{1 / p},
$$

then by the Radon-Nikodym theorem we have $h_{f}=h_{f}^{-} \mu$-almost everywhere in $X$ and by inequality (14) we also know that $\nu_{f} \ll \mu$. Moreover, in this case, from [HKT, Theorem 1.1(2)], we have $\nu_{f}(B) \geq C^{-1} \int_{B} g_{f}^{p} d \mu$ for the minimal $p$-weak upper gradient (in $\left.C_{q} \lambda B\right) g_{f}$ of $f$. From the above discussion, we see that if in addition to the hypotheses of Theorem 4.1 and Condition $r\left(V_{p}\right)$, if $a_{f}$ is associated with a Radon measure $\nu_{f}$ as in [HKT], then

$$
a_{f}(B) \approx \operatorname{rad}(B)\left(f_{B} h_{f}^{p} d \mu\right)^{1 / p}
$$

with $C h_{f}$ a $p$-weak upper gradient of $f$. Given that by the proof of Theorem 4.1, for continuous upper gradients $g$ of $f$ we also have $\nu_{f}(B) \leq C \int_{B} g^{p} d \mu$, it would be natural to ask whether the function $h_{f}=h_{f}^{-}$, obtained above, is comparable to a $p$-weak upper gradient of $f$. From the results of [HKT] we know that $h_{f} \geq C^{-1} g_{f}$ when $g_{f}$ is the minimal $p$-weak upper gradient of $f$, and so $C h_{f}$ is a $p$-weak upper gradient of $f$, but it may not be equivalent to the minimal $p$-weak upper gradient. So far we do not know the complete answer to this question. However, given that 
Condition $r\left(V_{p}\right)$ is automatically satisfied if $a_{f}$ is given in the form of (16), we have the following result.

Proposition 4.4. Under the hypotheses of Theorem 4.1, if the functional $f \mapsto a_{f}$ is given by a Radon measure $\nu_{f}$ as in (16), then

$$
a_{f}(B) \approx \operatorname{rad}(B)\left(f_{B} h_{f}^{p} d \mu\right)^{1 / p},
$$

where $h_{f}$ is given by the formula (11) and $h_{f}$ is comparable to some weak upper gradient of $f$.

The following theorem demonstrates that Condition $\left(V_{p}\right)$ of Definition 3.2 is necessary in order to obtain $p$-Poincaré inequality for some finite $p \geq 1$, for without this condition we merely have $\infty$-Poincaré inequality, which in turn does not imply a $p$-Poincaré inequality for any finite $p \geq 1$; see [DSW]. Furthermore, without Conditions $\left(V_{p}\right)$ and (iii) of Definition 3.2, the functional given by (5)

$$
a_{f}(B):=\left(f_{B} f_{B}|f(y)-f(x)|^{p} d \mu(y) d \mu(x)\right)^{1 / p}
$$

would satisfy the rest of Definition 3.2 (and would even satisfy Condition (iii) if $X$ is $\infty$-thick quasiconvex), but does not represent first-order calculus. Therefore it would seem that both Conditions $\left(V_{p}\right)$ and (iii) of Definition 3.2 are needed in order to obtain a functional associated with first-order calculus of the Newton-Sobolev spaces, which then in addition yields an improvement of the $\infty$-Poincaré inequality.

Theorem 4.5. Let $(X, d, \mu)$ be a complete metric space with $\mu$ a doubling measure. Then $X$ supports a $\infty$-Poincaré inequality if and only if $X$ supports a first order Poincaré inequality with $\mathcal{F}=N^{1, \infty}(X)$ and $f \mapsto a_{f}$ satisfies Condition (iii) of Definition 3.2.

We do not have a priori Lipschitz continuity (or any kind of continuity at all) of functions in $N^{1, \infty}(X)$, and so the proof of the above theorem is a bit more involved. Furthermore, even if the function $f$ happens to be Lipschitz continuous, its upper gradient need not be minorized by $\operatorname{Lip}(f)$. Even knowing that $X$ is quasiconvex does not give us direct control of the function $f$ constructed in the proof below.

Proof. Suppose that $(X, d, \mu)$ supports an $\infty$-Poincaré inequality. Then there are constants $\lambda, C>0$ such that when $f: X \rightarrow \mathbf{R} \cup\{-\infty, \infty\}$ is a measurable function and $g: X \rightarrow[0, \infty]$ is an upper gradient of $f$,

$$
f_{B}\left|f-f_{B}\right| d \mu \leq C \operatorname{rad}(B)\|g\|_{L^{\infty}(\lambda B)}
$$

for each ball $B \subset X$. Now, for each function $f \in N^{1, \infty}(X)$, define the functional $a_{f}: \mathcal{B} \rightarrow[0, \infty)$ by

$$
a_{f}(B)=C \inf _{g} \operatorname{rad}(B)\|g\|_{L^{\infty}(\lambda B)},
$$

where the infimum is over all non-negative Borel functions $g$ that are upper gradients of $f$ in $X$. With this choice of $a_{f}$, the Conditions (i), (ii) and (iii) of Definition 3.2 hold with the choice of $\mathcal{F}=N^{1, \infty}(X)$.

On the other hand, let us assume that $X$ supports a first order Poincaré inequality with an associated functional for which Condition (iii) of Definition 3.2 holds. One 
has to prove that $X$ supports a $\infty$-Poincaré inequality for functions in $N^{1, \infty}(X)$; this is equivalent, by [DJS, Theorem 4.7], to proving that $X$ is $\infty$-thick-quasiconvex as in Definition 2.4.

Let $x, y \in X$ such that $x \neq y, 0<\varepsilon<d(x, y) / 4$, and let $E \subset B(x, \varepsilon), F \subset B(y, \varepsilon)$ be measurable sets such that $\mu(E) \mu(F)>0$. Fix $n \in \mathbf{N}$ and let $\Gamma_{n}$ be the collection of all rectifiable paths connecting $E$ to $F$ such that $\ell(\gamma) \leq n d(x, y)$. Observe that by the choice of $\varepsilon$, if $p, q$ are the end points of $\gamma$, then $d(p, q) / 4 \leq d(x, y) \leq 4 d(p, q)$.

Suppose that $\operatorname{Mod}_{\infty}\left(\Gamma_{n}\right)=0$. By [DJ, Lemma 5.7] there exists a non-negative Borel measurable function $g \in L^{\infty}(X)$ such that $\|g\|_{L^{\infty}(X)}=0$ and for all $\gamma \in \Gamma_{n}$, the path integral $\int_{\gamma} g d s=\infty$. In this case we define

$$
f(z)=\inf _{\gamma \text { connecting } z \text { to } E} \int_{\gamma}(1+g) d s .
$$

Observe that $\|1+g\|_{L^{\infty}(X)}=1$ and $f=0$ on $E$. If $z \in F$ and $\gamma$ is a rectifiable path connecting $z$ to $E$, then either $\gamma \in \Gamma_{n}$ in which case $\int_{\gamma}(1+g) d s \geq \int_{\gamma} g d s=\infty$, or else $\gamma \notin \Gamma_{n}$, in which case $\ell(\gamma)>n d(x, y)$ and so $\int_{\gamma}(1+g) d s \geq \int_{\gamma} 1 d s>$ $n d(x, y)$. It follows that when $z \in F$ we have $f(z) \geq n d(x, y)$. Thus the function $\tilde{f}=\min \{f, 2 n d(x, y)\}$ has the properties that

(1) $\tilde{f}=0$ on $E$,

(2) $\tilde{f} \geq n d(x, y)$ on $F$,

(3) $\tilde{f} \in N^{1, \infty}(X)$,

(4) $1+g$ is an upper gradient of $\tilde{f}$ on $X$ with $\|1+g\|_{L^{\infty}(X)}=1$.

Let $y_{0} \in F$ and $x_{0} \in E$ be Lebesgue points of $\tilde{f}$. Such points exists by the Lebesgue differentiation theorem because $\mu(E) \mu(F)>0$. By using the chain of balls defined by $B_{i}=B\left(x_{0}, 2^{1-i} d(x, y)\right)$ if $i \geq 0$ and $B_{i}=B\left(y_{0}, 2^{1+i} d(x, y)\right)$ if $i \leq-1$, and using the first order Poincaré inequality with Condition (iii), we get

$$
\begin{aligned}
n \frac{d(x, y)}{2} & \leq n d\left(x_{0}, y_{0}\right) \leq \tilde{f}\left(y_{0}\right)=\left|\tilde{f}\left(x_{0}\right)-\tilde{f}\left(y_{0}\right)\right| \leq \sum_{i \in \mathbf{Z}}\left|\tilde{f}_{B_{i}}-\tilde{f}_{B_{i+1}}\right| \\
& \leq C \sum_{i \in \mathbf{Z}} \int_{B_{i}}\left|\tilde{f}-\tilde{f}_{B_{i}}\right| d \mu \leq C \sum_{i \in \mathbf{Z}} a_{\tilde{f}}\left(B_{i}\right) \leq C \sum_{i \in \mathbf{Z}} C \operatorname{rad}\left(B_{i}\right) \\
& \leq C \sum_{i \in \mathbf{Z}} 2^{-|i|} d(x, y)=C d(x, y) .
\end{aligned}
$$

Thus we must have $n \leq C$, with $C$ depending solely on the doubling constant and the constant of the Poincare inequality. Hence if $n>C$ then the path family $\Gamma_{n}$ must have positive $\infty$-Modulus, completing the proof of the theorem.

Given $1 \leq p<\infty$, it is a result of Keith [Ke3] that to verify $p$-Poincaré inequality for all functions in $N^{1, p}(X)$, it suffices to verify the inequality for Lipschitz functions and their upper gradients $\operatorname{Lip} f$, or equivalently, to verify the inequality for Lipschitz functions and their continuous upper gradients. The key tool in the proof of this fact is the Vitali-Carathéodory theorem which tells us that we can approximate non-negative functions in $L^{p}(X)$ from above with lower semicontinuous functions. Unfortunately, for $p=\infty$, such a theorem does not hold true, and so we do not know that verifying $\infty$-Poincaré inequality for Lipschitz functions and its upper gradient Lip $f$ in turn implies that $\infty$-Poincaré inequality holds for all $f \in N^{1, \infty}(X)$. On 
the other hand, the Sierpinski carpet supports an $\infty$-Poincaré inequality for locally Lipschitz continuous functions with continuous upper gradients but does not support an $\infty$-Poincaré inequality for functions in $N^{1, \infty}(X)$ (see [DJS, Corollary 4.15]). Hence we do not know whether the above Theorem 4.5 holds if we replace $\mathcal{F}=N^{1, \infty}(X)$ with $\mathcal{F}=\operatorname{LIP}^{\infty}(X)$. Indeed, as noted in [DJS] and [DSW], working with $\infty$-Poincaré inequality, while giving surprisingly elegant connections with the geometry of $X$, is difficult because of the lack of a Vitali-Carathéodory theorem and the non-local nature of the $L^{\infty}$-norm.

We finally point out that between the zero-th order Poincaré type inequality of [FPW] and the first order Poincaré inequality considered in [HKT] and this note, there is a wide class of "fractional order" Poincaré inequalities associated with Besov spaces; such Besov-Poincaré type inequalities are considered in [GKZ]. It would be interesting to know what conditions on the BMO-Poincaré type inequalities of [FPW] would be related to such a fractional order calculus of Besov spaces.

\section{References}

[ABKY] Aalto, D., L. Berkovits, O. E. Kansanen, and H. Yue: John-Nirenberg lemmas for a doubling measure. - Studia Math. 204, 2011, 21-37.

[BJM] BAdr, N., A. Jiménez-Del-Toro, and J. M. MARTELl: $L^{p}$ self-improvement of generalized Poincaré inequalities in spaces of homogeneous type. - J. Funct. Anal. 260, 2011, $3147-3188$.

[CW] ChuA, S.-K., and R. L. Wheeden: Self-improving properties of inequalities of Poincaré type on $s$-John domains. - Pacific J. Math. 250, 2011, 67-108.

[DJ] Durand-Cartagena, E., and J. A. Jaramillo: Pointwise Lipschitz functions on metric spaces. - J. Math. Anal. Appl. 363, 2010, 525-548.

[DJS] Durand-Cartagena, E., J.A. Jaramillo, and N. Shanmugalingam: The $\infty$ Poincaré inequality in metric measure spaces. - Michigan Math. J. 61, 2012, 63-85.

[DSW] Durand-Cartagena, E., N. Shanmugalingam, and A. Williams: p-Poincaré inequality vs. $\infty$-Poincaré inequality; some counter-examples. - Math. Z. 271, 2012, 447-467.

[FPW] Franchi, B., C. PÉrez, and R. L. Wheeden: Self-improving properties of JohnNirenberg and Poincaré inequalities on spaces of homogeneus type. - J. Funct. Anal. 153, 1998, 108-146.

[FW] Franchi, B., and R. L. Wheeden: Some remarks about Poincaré type inequalities and representation formulas in metric spaces of homogeneous type. - J. Inequal. Appl. 3, 1999, 65-89.

[GKS] Gogatishvili, A., P. Koskela, and N. Shanmugalingam: Interpolation properties of Besov spaces defined on metric spaces. - Math. Nachr. 283, 2010, 215-231.

[GKZ] Gogatishvili, A., P. Koskela, and Y. Zhou: Characterizations of Besov and TriebelLizorkin spaces on metric measure spaces. - Forum Math. (to appear).

[Ha] HajŁasz, P.: Sobolev spaces on metric-measure spaces. - In: Heat kernels and analysis on manifolds, graphs, and metric spaces (Paris), Contemp. Math. 338, Amer. Math. Soc., Providence, RI, 2002, 173-218.

[HaK] HajŁasz, P., and P. Koskela: Sobolev met Poincaré. - Mem. Amer. Math. Soc. 145:688, 2000 .

[HKT] Heikkinen, T., P. Koskela, and H. Tuominen: Sobolev-type spaces from generalized Poincaré inequalities. - Studia Math. 181, 2007, 1-16.

[He] Heinonen, J.: Lectures on analysis on metric spaces. - Springer, 2001. 
[HeKo] Heinonen, J., and P. Koskela: Quasiconformal maps in metric spaces with controlled geometry. - Acta Math. 181, 1998, 1-61.

[HKST] Heinonen, J., P. Koskela, N. Shanmugalingam, and J. Tyson: Sobolev spaces on metric measure spaces - an approach based on upper gradients. - In preparation.

[JJRRS] JÄRVEnpÄÄ, E., M. JÄRVEnpÄÄ, K. Rogovin, S. Rogovin, and N. Shanmugalingam: Measurability of equivalence classes and $M E C_{p}$-property in metric spaces. - Rev. Mat. Iberoam. 23, 2007, 1135-1153.

[Ke3] KeIth, S.: Modulus and the Poincaré inequality on metric measure spaces. - Math. Z. $245,2003,255-292$.

[KR] Keith, S., and K. Rajala: A remark on Poincaré inequalities on metric measure spaces. - Math. Scand. 9:5, 2004, 299-304.

[KZ KeIth, S., and X. Zhong: The Poincaré inequality is an open ended condition. - Ann. of Math. (2) 167, 2008, 575-599.

[Ko] Korte, R.: Geometric implications of the Poincaré inequality. - Licentiate's thesis, Helsinki Univ. of Technology, 2006.

[KoMc] Koskela, P., and P. McManus: Quasiconformal mappings and Sobolev spaces. - Studia Math. 131, 1998, 1-17.

[KST] Koskela, P., N. Shanmugalingam, and J. Tyson: Dirichlet forms, Poincaré inequalities, and the Sobolev spaces of Korevaar and Schoen. - Potential Anal. 21:3, 2004, 241-262.

[LP] Lerner, A., and C. PÉrez: Self-improving properties of generalized Poincaré type inequalities through rearrangements. - Math. Scand. 97, 2006, 217-234.

[MP1] Macmanus, P., and C. PÉrez: Generalized Poincaré inequalities: Sharp self-improving properties. - Int. Math. Res. Not. IMRN 2, 1998, 101-116.

[MP2] MacManus, P., and C. Pérez: Trudinger's inequality without derivatives. - Trans. Amer. Math. Soc. 354, 2002, 1997-2012.

[OP] Orobitg, J., and C. PÉrez: $A_{p}$ weights for nondoubling measures in $\mathbf{R}^{n}$ and applications. - Trans. Amer. Math. Soc. 354, 2002, 2013-2033.

[S-C] Saloff-Coste, L.: Aspects of Sobolev-type inequalities. - London Math. Soc. Lecture Note Ser. 289, Cambridge Univ. Press, Cambridge, 2002.

[Se] Semmes, S.: Finding curves on general spaces through quantitative topology, with applications to Sobolev and Poincaré inequalities. - Selecta Math. (N.S.) 2, 1996, 155-295.

[Sh1] Shanmugalingam, N.: Newtonian spaces: An extension of Sobolev spaces to metric measure spaces. - Ph.D. Thesis, Univ. of Michigan, 1999, http://math.uc.edu/ nages/ papers.html.

[Sh] Shanmugalingam, N.: Newtonian spaces: An extension of Sobolev spaces to metric measure spaces. - Rev. Mat. Iberoam. 16, 2000, 243-279.

[Sh2] Shanmugalingam, N.: A universality property of Sobolev spaces in metric measure spaces. - In: Sobolev spaces in mathematics. I, Int. Math. Ser. (N. Y.) 8, Springer, New York, 2009, 345-359.

[Va] VÄISÄLÄ, J.: Lectures on $n$-dimensional quasiconformal mappings. - Lecture Notes in Math. 229, Springer-Verlag, Berlin, 1971. 